\title{
Entrelacs
}

Cinéma et audiovisuel

Hors-série $n^{\circ} 4$ | 2016

Paysages en séries

\section{The Big Empty : représentation(s) de l'espace interstellaire à la télévision}

The Big Empty

Florent Favard

\section{OpenEdition}

Journals

Édition électronique

URL : http://journals.openedition.org/entrelacs/2148

DOI : $10.4000 /$ entrelacs. 2148

ISBN : $2261-5482$

ISSN : 2261-5482

Éditeur

Éditions Téraèdre

Édition imprimée

Date de publication : 1 novembre 2016

ISSN : 1266-7188

\section{Référence électronique}

Florent Favard, «The Big Empty : représentation(s) de l'espace interstellaire à la télévision », Entrelacs

[En ligne], Hors-série n 4 | 2016, mis en ligne le 06 février 2018, consulté le 19 avril 2019. URL : http:// journals.openedition.org/entrelacs/2148; DOI : 10.4000/entrelacs.2148

Ce document a été généré automatiquement le 19 avril 2019

Tous droits réservés 


\section{The Big Empty : représentation(s) de l'espace interstellaire à la télévision}

The Big Empty

Florent Favard

Space, the final frontier.

2 Ces mots ouvrent le générique de la série culte Star Trek (NBC, 1966-1969), mettant en scène le vaisseau Enterprise et son équipage, engagés dans une mission d'exploration de la galaxie. Ils incarnent à eux seuls l'exploration spatiale, fictionnelle comme réelle, une exploration renouvelée par les médiums audiovisuels que sont le cinéma et la télévision. Ainsi que le souligne par exemple J.P. Telotte, les séries télévisées sont peut-être bien aujourd'hui le lieu où la science-fiction peut viser de nouveaux horizons, se réinventer (Telotte, 2008); si elles ont longtemps subi la concurrence du cinéma et de ses effets spéciaux aux lourds moyens, les séries science-fictionnelles ont su trouver leur propre identité et, parfois même, s'emparer d'un sous-genre pour le porter plus loin que ne l'aurait fait le grand écran.

3 Les séries de space opera sont de celles-là. Sous-genre déjà « usé » avant que n'apparaisse la télévision, il a trouvé ses heures de gloire dans les pulps, ces magazines peu onéreux rassemblant bandes dessinées et nouvelles, puis dans les serials cinématographiques, ces «films découpés en épisodes» (Dufour, 2011: 38) diffusés de façon hebdomadaire, anticipant déjà sur la série télévisée comme forme audiovisuelle. Le space opera, si l'on en croit l'écrivain William Tucker qui lui a donné son nom, met en scène des aventures à travers une galaxie souvent très peuplée, le planet opera en étant la variation à l'échelle d'un système solaire, voire d'une seule planète - du moment que les personnages maitrisent le voyage spatial. Lorsque la télévision états-unienne s'en est emparée, elle a d'abord strictement suivi les codes du serial, à l'image du passage de Flash Gordon du grand (Frederick Stephani, Ray Taylor, 1936) au petit écran (Syndication, 1954-1955) : aux décors en carton-pâte s'imbriquaient des intrigues manichéennes «marqué[es] par le climat social de l'époque » (Dixon, 2008 : 93), en pleine Guerre Froide. C'est avec Star Trek que le sous-genre va connaître un renouveau: des personnages moins lisses, un vaste 
monde fictionnel en nuance de gris, et un public féminin au rendez-vous (Jenkins, 2013 : 185). Sous l'égide de la licence Star Trek, qui totalise six séries à ce jour, le space opera télévisuel va dominer la télévision anglo-saxonne, surtout durant son âge d'or des années 1980-2000, porté par les succès cinématographiques des films Star Trek et Star Wars. Les effets spéciaux deviennent numériques - surtout avec la révolution qu'incarne Babylon 5 (PTEN>TNT, 1993-1998) -, l'image passe en 16/9ème, et les écrans eux-mêmes deviennent démesurés : les séries de space opera rivalisent aujourd'hui avec les films lorsqu'il s'agit de montrer l'espace interstellaire.

4 Car c'est bien de cela qu'il sera question ici : la représentation de l'espace interstellaire, dans une perspective essentiellement formaliste. Beaucoup d'encre a coulé sur la portée politique et socio-culturelle des séries de science-fiction; de nombreuses plumes se sont attardées sur leurs innovations technologiques et leur complexité narrative. Mais peu s'interrogent sur l'esthétique de la représentation de l'espace interstellaire, encore moins dans une perspective sérielle. Pourtant la question est fascinante puisque les mondes fictionnels des séries de space opera sont presque tout entiers bâtis sur des éléments qui ont peu, ou pas, de correspondants dans le monde réel, ainsi que le dirait Thomas Pavel (1988: 76) : ces mondes fictionnels sont en "porte-à-faux" par rapport au nôtre, explorant des régions dont l'immense majorité des êtres humains ne fera jamais l'expérience. À l'heure où j'écris ces lignes, six personnes se trouvent dans l'espace, à bord de la Station Spatiale Internationale ; l'espace étant défini par ce qui se trouve audelà de la Ligne Kármán, c'est-à-dire au-delà de $100 \mathrm{~km}$ d'altitude (selon la Fédération Aéronautique Internationale). 547 personnes ont passé cette limite dans l'histoire de l'humanité, et aucune n'a jamais dépassé le voisinage de la Lune. Seules nos sondes robotisées ont exploré d'autres planètes (notamment les surfaces de Mars et de Vénus) et tout juste dépassé les limites du système solaire: Voyager 1, lancée en 1977, se trouve à plus de 18 milliards de kilomètres du Soleil et évolue à $17 \mathrm{~km} / \mathrm{s}$. Tout ce que nous savons des astres les plus distants découle d'observations menées depuis la Terre. À défaut d'une expérience empirique de ces environnements lointains, nous les expérimentons à travers la (science-)fiction, qui peuple nos imaginaires de vaisseaux à vitesse supraluminique et de planètes à la vie abondante, abritant des civilisations extraterrestres. Mais pour atteindre ces astres lointains, encore faut-il traverser l'espace interstellaire et une galaxie dont les dimensions dépassent toute capacité d'appréhension par l'esprit humain.

C'est à cet espace que se consacre le présent article; non pas aux planètes, qui sont souvent des chimères construites à partir de la nôtre (que les scènes soient tournées sur Terre ou conçues numériquement), mais au vide interstellaire. Il n'existe pas vraiment, à ce jour, d'appareil théorique esthétique capable d'appréhender cet espace vide, dont la physique nous apprend qu'il n'a ni haut, ni bas, et que tout euclidien qu'il paraisse, il peut être «tordu» par des forces gravitationnelles. Un bon point de départ semble être la notion de paysage qui, comme l'explique Anne Cauquelin, est née avec la perspective au $\mathrm{XV}^{\mathrm{e}}$ siècle : le paysage n'est pas la nature elle-même et, même en dehors du tableau (et aujourd'hui, de l'écran), il reste une construction située, une «forme symbolique » qui « lie [...] la parole, les sensibilités, les actes, dans un même dispositif » (2007:29-30). À cette différence près que, si l'espace interstellaire peut être considéré comme le dernier endroit « naturel », non transformé par l'activité humaine, il est aussi un espace que très peu d'humains ont visité. Tout ce que nous expérimentons de l'espace interstellaire passe par le biais de l'imagination et de la fiction, quand bien même les lois physiques permettent d'extrapoler ce que serait une expérience empirique du voyage interstellaire. 
Ce vide infini est peut-être le seul à correspondre à la notion de «non-lieu » selon MarcAugé, c'est-à-dire un espace, une "étendue abstraite » (1992: 105), sans histoire, sans identité, sans relation à un autre lieu ; mais nous verrons là aussi que les choses ne sont pas si évidentes.

6 Loin de proposer une analyse exhaustive de l'espace interstellaire dans les séries télévisées, je propose ici un premier défrichement de ses particularités, qui le distinguent ou le rapprochent de sa représentation au cinéma. Ce tour d'horizon se fera en quatre temps, et explorera la riche production anglo-saxonne dans le domaine du space opera, en partant de l'échelle la plus familière - celle de l'humain - pour approcher petit à petit les échelles les plus cosmiques, et donner, je l'espère, matière à creuser le sujet quelques années-lumière de plus.

\section{L'être humain dans le vide spatial}

7 Cette première catégorie concerne les scènes dans lesquelles un personnage, sinon humain, du moins humanoïde ou anthropomorphisé, se retrouve confronté au vide spatial sans aucune autre protection qu'une éventuelle tenue adaptée à une sortie extravéhiculaire ; c'est-à-dire, lorsque ce personnage est en dehors du cocon protecteur qu'est le vaisseau spatial et que, le cas échéant, la proximité de celui-ci n'influe pas de manière significative sur les forces physiques qui s'exercent sur le sujet. Ce dernier est donc en état d'apesanteur : il ne subit pas, ou très peu, les effets de la pesanteur mais sa trajectoire peut lui faire courir le risque d'être attiré par un corps massif - par exemple, une planète.

Ces situations sont loin d'être uniquement science-fictionnelles puisque des astronautes les expérimentent régulièrement en orbite; elles incarnent l'essence même du sublime kantien. Le lien entre sublime et science-fiction, initialement exploré par Cornel Robu (1988), est décrit au cinéma par Éric Dufour comme « constitutif de l'image du film de S$\mathrm{F}$ » autant que de la narration dans un sens large. Dufour distingue ainsi, comme le faisait Robu, le sublime mathématique, celui des dimensions, de l'infini, du sublime dynamique, celui de l'effroi, du péril (2011: 151-152).

9 Un personnage flottant dans le vide est théoriquement confronté au sublime mathématique, la différence d'échelle étant maximale entre le corps humain, repère familier des spectateur.ice.s, et l'immensité de l'espace interstellaire. Mais le petit écran de la télévision a longtemps peiné à rendre cette impression d'infini : parce que l'infini rend beaucoup mieux au cinéma, et ce dès les révolutions visuelles que sont 2001, A Space Odyssey (Stanley Kubrick, 1968) et Star Wars Episode IV (George Lucas, 1977); et parce que les budgets réduits des séries, avant l'utilisation massive des images de synthèse, ne permettent pas de donner aux interprètes la vraie liberté de mouvement permise en apesanteur. En 1997 par exemple, dans Star Trek: Voyager (UPN, 1995-2001), B’Elanna et Tom passent une bonne partie de l'épisode Day of Honor (S04E03) perdu.e.s dans l'espace, leurs combinaisons perdant peu à peu de l'oxygène. Typique de ce genre de scène, l'épisode passe vite sur un plan d'ensemble pour se focaliser sur des plans serrés, et troque le sublime mathématique pour le sublime dynamique: le manque d'oxygène ramène l'hostilité de l'espace interstellaire sur le devant de la scène.

Mais loin du statisme des naufragé.e.s de l'espace, la sortie extravéhiculaire est souvent l'occasion d'une fuite en avant du corps dans l'espace, sur une trajectoire rectiligne et 
sans aucun ralentissement. Là où les premières séries de science-fiction montraient surtout des corps s'éloignant d'un sas, rétrécissant dans le cadre à mesure qu'ils s'enfoncent dans la profondeur du champ, les séries contemporaines ont là encore tiré parti des images de synthèse pour suivre le corps, vivant ou mort, le long de sa trajectoire, offrant à voir des scènes spectaculaires qui permettent de mieux appréhender l'espace. Ce sont notamment les fréquentes exécutions dans Battlestar Galactica (Syfy, 2003-2009), via les sas des vaisseaux spatiaux, qui donnent lieu à l'expression « to airlock someone ». La caméra suit le corps derrière lequel défilent les étoiles ou la paroi du vaisseau, le flou de mouvement achevant de donner l'impression de vitesse tandis que les recadrages nerveux enserrent la victime comme dans un documentaire animalier. Même son de cloche chez The 100 (The CW, 2014-présent), héritière de Battlestar Galactica, qui elle aussi fait du sas une méthode d'exécution et suit les corps projetés dans le vide, à la différence près que sa mise en scène est moins documentaire, donnant des scènes de facture plus classique mais tout aussi épiques. Non sans ironie, la dernière sortie extravéhiculaire de la série à ce jour (dans le présent de la narration) est volontaire : dans l'épisode Inclement Weather (S02E02), Jaha, restée derrière après que les survivant.e.s sont descendu.e.s sur Terre, entend se projeter d'un bout à l'autre d'une station orbitale circulaire pour atteindre un moyen de transport, et parcourt son diamètre à grande vitesse. La scène mélange les échelles de plan, des plus serrées (la fente sur la visière du casque) aux plus lointaines, et les plans mélangent le corps - qui n'est souvent plus qu'un point blanc -, le vaisseau, la planète loin derrière et le vide intersidéral.

11 L'espace n'est pas toujours un lieu hostile pour la figure humaine : il devient familier dans Battlestar Galactica, où le privilège ultime est d'avoir une cabine " avec vue », c'est-à-dire, avec un hublot. Dans The Expanse (Syfy, 2015-présent), autre héritière de Battlestar Galactica, l'espace interstellaire est représenté de façon si réaliste et banale qu'on peut y voir, dans l'épisode Rock Bottom (S01E06), un mineur d'astéroïde relever nonchalamment sa visière, expirer, et enlever un fil lâche qui le gênait avant de refermer son casque. La mise en scène non-spectaculaire de son geste véhicule la surprise puisqu'elle transgresse les représentations de l'espace comme un milieu instantanément hostile à la vie (en réalité, les corps n'y explosent pas sous l'absence de pression, pas plus qu'ils ne gèlent en quelques secondes: les radiations sont plus à craindre). Farscape (Nine Network, 1999-2003), dont le ton comique et réflexif en fait un Las Vegas Parano dans l'espace, pousse aussi cette transgression assez loin en ce que la plupart des personnages font au moins une fois l'expérience d'une sortie extravéhiculaire non-protégée: si Crichton, l'humain, peut virevolter plusieurs secondes dans Look at the Princess, Part 2 (S02E11), D'Argo, le Luxan, peut survivre un quart d'heure dans le vide spatial, par exemple dans They've Got a Secret (S01E10) : la série peut alors, au gré des épisodes, jongler avec deux visions de l'espace, l'une le codant comme hostile, l'autre comme fantaisiste et source d'acrobaties incroyables.

12 La série Doctor Who (BBC1, 1963-1989; 2005-présent), conçue pour être intergénérationnelle, porte sur le vide interstellaire un regard souvent attendri et sublimé. Un des points nodaux de la série - c'est-à-dire, un des ingrédients de la formule qui structure les épisodes (Esquenazi, 2010:105) - se cristallise autour du vaisseau spatiotemporel utilisé par le personnage éponyme, vaisseau qui peut déployer une bulle d'air protectrice lorsque ses portes sont ouvertes dans le vide, permettant alors aux personnages, souvent habillés d'une tenue qui n'a rien de science-fictionnelle (citadine, généralement), d'admirer le vide interstellaire et ses formations naturelles, nébuleuses, 
étoiles et galaxies, sans aucune frontière. Le Docteur montre ainsi à Donna la formation de la Terre dans The Runaway Bride (S03X01), fait flotter Amy dans l'espace - alors qu'elle est en robe de chambre et pyjama - en la tenant par le pied dans The Beast Below (S05E02), et reçoit du « courrier » sur la forme d'une petite boîte lumineuse qui vient frapper à sa porte au beau milieu de l'espace dans The Doctor's Wife (S06E02). Endroit critique du vaisseau qui ouvre toujours sur un nouveau lieu extraordinaire, les portes de la salle de pilotage dévoilent aussi l'infini spatial sans jamais le coder comme hostile, laissant ainsi les personnages, en plans serrés, s'émerveiller à sa vue.

\section{Océan spatial}

13 De la figure humaine au vaisseau spatial, il n'y a qu'un pas, que les protagonistes franchissent souvent lorsqu'il s'agit d'évoluer sur la coque du vaisseau. Ces sorties extravéhiculaires à la surface des véhicules souvent démesurés sont intéressantes en ce qu'elles transgressent les plans souvent lointains sur ces derniers, que les séries recyclent au fil des épisodes : soudain, un vaisseau qui occupait à peine la partie centrale de l'écran devient méconnaissable alors que notre perspective change - et j'entends ici perspective comme point de vue, mais aussi comme règles de représentation. Le vaisseau Destiny de Stargate Universe (Syfy, 2009-2011) est en cela fascinant grâce à sa forme atypique, plat, triangulaire avec des côtés concaves et une poupe convexe. Lorsque les protagonistes doivent évoluer à sa surface, sur sa tranche, dans Incursion, Part 2 (S01E20), les lignes de fuites sont très prononcées, creusant la profondeur du champ et faisant apparaître l'appareil comme démesuré. L'effet de défamiliarisation est alors maximal, puisque la figure humaine nous pousse soudain à réinterpréter notre perception de cet élément qui paraissait familier, de par ses apparitions régulières.

Ces dernières sont d'autant plus familières qu'elles sont toujours filmées selon un modèle standard et codé qui, s'il n'a pas été instauré par Star Trek, a certainement été cristallisé par cette licence dont les nombreuses séries ont considérablement pesé sur l'imaginaire collectif : ce modèle, c'est celui de l'Enterprise naviguant en ligne droite, souvent de la gauche vers la droite, et croisant d'autres vaisseaux invariablement situés sur le même plan, et si proches comparé à la vastitude de l'espace interstellaire que cela en est presque risible. Mais il faut bien comprendre que l'approche standard est liée à des contraintes économiques (trop peu de budget dans les séries du siècle dernier pour faire des plans originaux et complexes sur maquettes, d'autant que des centaines de ces plans doivent être tournés), technologiques (sur le petit écran, il faut montrer les deux vaisseaux dans le même plan sans qu'ils ne soient deux points illisibles) et esthétiques (le poids de ces conventions tout à fait fictionnelles n'a d'égal que celui que nous faisons peser sur les paysages terrestres).

15 Il a fallu attendre la révolution numérique, des écrans plus grands, le format $16 / 9^{\mathrm{e}}$ ainsi que la volonté de transgresser certains codes pour voir apparaître des séries cherchant à renouveler la façon de montrer l'espace interstellaire. Les batailles spatiales sont un moyen idéal de creuser la perspective puisqu'elles impliquent de nombreux vaisseaux. Dès les années 1990, Babylon 5, pionnière de l'utilisation d'effets spéciaux numériques à la télévision, compose de riches plans où des essaims de vaisseaux de guerre livrent bataille sur des fonds complexes, souvent colorés par des nébuleuses et des planètes (JohnsonSmith, 2005 : 237-238). 
Des séries comme Star Trek: Deep Space Nine (Syndication, 1993-1999) et Battlestar Galactica profitent des batailles spatiales pour casser le modèle planaire, linéaire, et explorer l'espace dans ses trois dimensions. La seconde, notamment, emploie les tirs, les effets de particules des explosions, les essaims de petits vaisseaux et une plus grande distance entre les vaisseaux-mères pour creuser la profondeur du plan grâce à de fréquents recadrages et zooms. Cette liberté est d'abord technologique et, depuis Battlestar Galactica, ce nouveau modèle de mise en scène nerveuse ponctuée de zooms a été repris par Stargate Universe ou encore The Expanse. Cette dernière marque un nouveau pas dans l'exploration de l'espace (du plan) puisque ses standards de réalismes l'obligent à figurer les distances de façon plus adéquate : les vaisseaux sont très éloignés, les tirs de missiles génèrent un suspense qui dure plusieurs secondes, et les appareils frôlent la caméra à grande vitesse avant de disparaitre dans le lointain. Nul doute qu'à cette échelle, les évolutions technologiques - celles des effets spéciaux comme celles des fabricants de téléviseurs, toujours plus grands - permettent une représentation, sinon hyperréaliste, tout au moins à même de rivaliser avec le grand écran en termes de spectacle.

\section{L'espace interstellaire comme lieu du sublime}

17 Au-delà de l'échelle des vaisseaux et des structures artificielles, on trouve les structures naturelles que sont les planètes, étoiles et nébuleuses. Celles-ci peuvent servir de simples décors de fond, quand bien même les séries feuilletonnantes, "narrativement complexes» (Mittell, 2015) des années 1990 et suivantes ont su leur trouver une utilité. Babylon 5, série à l'intrigue dense, se sert des repères astraux pour créer, au-delà d'espaces génériques, des lieux reconnaissables qui deviennent chargés d'histoire (celle du monde fictionnel) et ne sont plus des «non-lieux ». La planète Sigma-957 et la nébuleuse pourpre sur laquelle elle se détache laissent une empreinte suffisante dans la mémoire de la spectatrice pour que l'utilisation récurrente de ce lieu dans Mind War (S01E06) et Voices of Authority (S03E05) en fasse un lieu à part; de même, Coriana VI et sa nébuleuse orangée, irisée de vert, sont un lieu-clé riche d'histoire, où se déroule une bataille centrale dans l'intrigue de la série (Into the Fire, S04E06) et où disparaît à jamais un des protagonistes dans l'épisode final, Sleeping in Light (S05E22).

La licence Star Trek, de son côté, a toujours été fascinée par les nébuleuses et autres astres démesurés qui peuvent confronter l'équipage de l'Enterprise au "sublime de la semaine » dans chaque épisode. Face à ces structures qui peuvent couvrir des milliards de kilomètres, se pose toutefois un problème de taille: les représenter via un plan d'ensemble de manière un tant soit peu réaliste, c'est souvent employer un point de vue similaire aux observations télescopiques, dont les objectifs écrasent toute perspective, tout volume. Explorer l'intérieur de la nébuleuse pose un problème similaire en ce que, de même que les planètes qui servent de toile de fond aux plans de ces séries, la nébuleuse vue de l'intérieur apparaît tout aussi plate, sans dimension et donc sans aucun sens du volume et de la matière de ces structures.

Star Trek: Voyager (UPN, 1995-2001) résout le problème indirectement, par exemple dans un épisode comme One (S04E25) : le vaisseau Voyager doit y traverser une gigantesque nébuleuse. Les plans à l'extérieur du vaisseau montrent d'abord la nébuleuse de loin : elle apparait sans volume. Puis le vaisseau y pénètre : le décor de fond est texturé, mais plat, et seul un voile uniforme colorant légèrement le vaisseau simule le nuage de gaz. C'est dans la salle d'observation du vaisseau, dotée d'un écran géant, qu'une représentation 
tridimensionnelle de la nébuleuse, assortie d'une grille, permet de mesurer l'avancée du vaisseau à l'intérieur du volume.

Battlestar Galactica parvient à proposer une représentation plus réaliste de la traversée d'une nébuleuse, en insistant, narrativement, sur le danger qui guette les humains (les radiations intenses) et via la mise en scène, sur la texture de cet espace sans commune mesure avec le vide interstellaire. À mesure que les vaisseaux se déplacent, les volutes de gaz en cachent certains, en découvrent d'autres, tandis que la forte lumière et le "souffle » perçu sur les coques des vaisseaux achèvent de faire de la nébuleuse un enfer. Là encore, le progrès des effets spéciaux numériques permet de donner corps à l'espace, de mesurer sa distance et la progression des protagonistes, et de rendre le sublime à la fois mathématique et dynamique qu'il est censé véhiculer.

Rares sont les séries qui se risquent à représenter les structures autrement plus grandes que sont les galaxies, et à s'intéresser à l'espace intergalactique, démesuré. Récemment, c'est la licence Stargate qui a exploré ces contrées encore inconnues. Stargate SG1 (Showtime>Syfy, 1997-2007) se déroule dans notre Voie Lactée, tandis que la série dérivée Stargate Atlantis (Syfy, 2004-2009) prend place dans la galaxie voisine d'Andromède ; les personnages construisent alors un "pont» entre les deux galaxies, une station-relais suspendue dans le vide intergalactique, donnant à voir les deux galaxies à distance vision rare puisqu'elles sont à des millions d'années-lumière l'une de l'autre - et s'imposant comme un lieu possédant une histoire et dans lequel des protagonistes se sont investis, de sa construction dans The Return, Part 1 (SGA, S03E10), à sa destruction dans Midway (SGA, S04E17). Stargate Universe pousse plus loin cette logique en ce qu'elle établit dès son épisode pilote que le vaisseau Destiny passe de galaxie en galaxie dans sa mission d'exploration millénaire, et ce via une représentation holographique. C'est dans Sabotage (S01E16) que cette logique d'exploration et d'expansion du monde fictionnel est actée, lors du premier bond du vaisseau entre deux galaxies. La série donne alors à voir des images rarement vues à la télévision ou même au cinéma, celle d'une galaxie dont on s'éloigne peu à peu, et dont chaque plan révèle alors le volume, la structure très détaillée ; une vision sublime - au sens kantien - que l'on retrouve aussi dans le travelling supraluminique à travers la galaxie, qui conclue la troisième saison de Battlestar Galactica. Mais Stargate Universe s'attache à donner une représentation réaliste des distances: chaque point lumineux aperçu dans l'épisode Sabotage n'est plus une étoile, mais, vu du vide intergalactique, une autre galaxie. La licence Stargate a ainsi participé à déployer le space opera selon de nouvelles modalités, en allant toujours plus loin, toujours plus vite.

\section{Hyperespaces et subspaces : à la frontière du représentable}

Pour aller si vite, les vaisseaux spatiaux dépassent souvent la vitesse de la lumière pour entrer dans ce que ces séries nomment communément l'hyperespace. Il est intéressant de noter que la représentation de cet espace distordu, où les lois de la physique telles que nous les connaissons n'ont plus court, est propre à chaque série ; le tunnel qu'on dirait dit de fumée de Doctor Who la distingue du plan au flux d'énergie rectiligne de Dark Matter (Space, 2015-présent), ou des étoiles « étirées » comme via un prisme de la licence Star Trek. Ces séquences de voyage hyperspatial maintes fois répétées, ouvrant souvent une scène comme le ferait un plan d'ensemble, participent de l'identité visuelle et sonore d'une série. 

irruptions dans ces programmes, notamment dans Star trek qui s'embarrasse rarement des lois de la physique et multiplie les subspaces comme si l'univers était constitué de couches successives. Mais à la différence de l'hyperespace qui doit être une signature visuelle propre à chaque série, les subspaces doivent sans cesse se démarquer de ce qui s'est fait avant, très souvent par la couleur. On y trouve des espaces sans plus aucun point de repère : le Null Space de Star Trek: The Next Generation (Syndication, 1987-1994) est un espace entièrement blanc qui supprime jusqu'à l'impression de profondeur elle-même dans The Outcast (S05E17). Dans Star Trek: Voyager le Fluidic Space, que les personnages visitent à plusieurs reprises durant la saison 4 , est de même un espace qui n'a aucune vocation à être exploré, et donc les nuances de jaune et de vert n'ont d'autre fonction que de connoter l'hostilité du lieu.

À l'opposé de ces espaces richement colorés mais jamais codés comme des lieux ou même des étendues explorables, se trouvent des espaces qui s'assument explicitement comme non-représentables car impossibles à appréhender par l'esprit humain. C'est notamment le cas du Q Continuum dans la licence Star Trek, que l'équipage du Voyager explore physiquement à deux reprises. Dans Death Wish (S02E18) les personnages découvrent pour la première fois ce plan d'existence où vivent les $Q$, des êtres omnipotents. Mais l'endroit leur apparaît sous la forme d'une aire de repos au bord d'une autoroute, au cœur du désert états-unien, un paysage pétri de conventions familières à mille années-lumière de l'espace interstellaire. Lorsque les $Q$ sont divisés par une guerre civile, c'est cette fois-ci un décor évoquant la Guerre de Sécession américaine que les humains découvrent en visitant le Continuum, dans The $Q$ and the Grey (S03E11). Alors même que cette guerre a un impact sur notre plan d'existence (des étoiles explosent à tout-va), c'est à coup de fusil que la guerre civile des $Q$ paraît se régler aux yeux des humains. La série Farscape emploie un procédé similaire lorsqu'à plusieurs reprises elle envoie l'astronaute Crichton au croisement de toutes les réalités possibles, dans un lieu habité par une entité reconnaissable, un homme en costume, et évoquant un iceberg sous une tempête de neige nocturne.

Il est intéressant de constater que cette façon de représenter des espaces incommensurables, tenant du sublime, par des lieux familiers, ou du moins des espaces plus facilement appréhendables par l'esprit humain, se retrouve dans des séries de fantasy comme Angel (The WB, 1999-2004), dont la White Room baignée de blanc se substitue à un plan supérieur: le récit va alors si loin qu'il emploie un procédé qui transgresse la frontière entre les genres.

\section{The Final Frontier}

Il y aurait encore beaucoup à dire sur la représentation de l'espace interstellaire sur les écrans de télévision, notamment sur la création du familier au sein d'un espace dont aucune expérience empirique ne peut vraiment rendre compte. J'espère avoir ici montré le potentiel des séries et la façon dont leurs modalités narratives et de représentation distinguent leur vision de l'espace du cinéma, quand dans le même temps elles en sont les héritières. Si les évolutions technologiques ont permis aux séries de rattraper le cinéma en termes de moyens, les premières ont auparavant construit leurs propres conventions, et ont su investir l'espace interstellaire pour y construire des lieux riches d'histoires, tout en normalisant des points de vue successifs, du classique Star Trek au réaliste Battlestar 
Galactica en passant par le subversif d'un Doctor Who. Reste à voir si la nouvelle vague du space opera télévisuel anglo-saxon, portée par The Expanse, Dark Matter, ou encore la prochaine série de la licence Star Trek, s'appuiera longtemps sur ces modèles, ou bien donnera à voir de nouveaux paysages interstellaires, un nouveau point de vue sur des espaces qu'il nous reste à voir de nos propres yeux.

\section{Bibliographie}

Augé, Marc. Non-lieux : Introduction à une anthropologie de la surmodernité. Paris : Seuil, 1992.

Cauquelin, Anne. L'Invention du paysage. Paris : PUF, 2000 (seconde édition, 2007).

Dixon, Wheeler Winston. « Tomorrow Land TV : The Space Opera and Early Science Fiction Television ", The Essential Science Fiction Television Reader. Telotte, J. P. Lexington : University Press of Kentucky, 2008. p. 93-110.

Dufour, Éric. Le Cinéma de science-fiction. Paris : Armand Colin, 2011.

Esquenazi, Jean-Pierre. Les Séries télévisées : L'Avenir du cinéma ? Paris : Armand Colin, 2010.

Jenkins, Henry. Textual Poachers: Television Fans and Participatory Culture. Londres : New York, Routledge, 1992 (édition révisée, 2013).

Mittell, Jason. Complex TV: The Poetics of Contemporary Television Storytelling. New York, Londres: New York University Press, 2015.

Pavel, Thomas. Univers de la fiction. Paris : Seuil, 1988.

Robu, Cornel. «A Key to Science Fiction : The Sublime. » Foundation \#42 (1988): pp. 21-37.

Telotte, J. P. The Essential Science Fiction Television Reader. Lexington : University Press of Kentucky, 2008.

\section{AUTEUR}

\section{FLORENT FAVARD}

Florent Favard est Docteur en arts, spécialise dans les études cinématographiques et audiovisuelles. Après avoir soutenu une thèse sur la complexité narrative des séries télévisées de science-fiction contemporaines, il poursuit actuellement ses recherches dans une perspective narratologique contextualiste et transmédiatique focalisée sur les genres de l'imaginaire. 